\title{
Validity and Reliability of the Obsession with the COVID-19 Scale
}

\section{ART ICLE INF O}

\section{Article Type}

Descriptive Study

\section{Authors}

Yaghoobi A. ${ }^{1} \mathrm{PhD}$,

Saeighi Mameghani Sh. ${ }^{2} P h D$,

Palancl M. ${ }^{3} P h D$

Karimi K.*1 $P h D$

How to cite this article
Yaghoobi A, Saeighi Mameghani
Sh, Palancl M, Karimi K. Validity
and Reliability of the Obsession
with the COVID-19 Scale. Iranian
Journal of War \& Public Health.
2020;12(4):235-240.

\section{A B S T R A C T}

Aims The spread of Covid-19 worldwide has challenged public health and has had various psychological consequences. The present study aimed to investigate the psychometric properties of the Obsession with the COVID-19 scale.

Instrument \& Methods As a descriptive-correlational study and to validate the Obsession with the COVID-19 scale, 392 people over 18 participated online, using the available sampling method from May to August 2020. A demographic questionnaire, Obsession with the COVID-19 Scale and Fear of Covid-19 Scale, was used to collect data. To evaluate the instrument's reliability, Cronbach's alpha internal consistency method and halving in the form of SPSS 25 statistical software and to evaluate the construct validity, confirmatory and exploratory factor analysis with the orthogonal rotation of Varimax type in Lisrel 8.8 software were used.

Findings Exploratory factor analysis identified all four items as one factor. Also, the results of the confirmatory factor analysis confirmed the construction. The convergent validity of the scale with fear of Covid-19 showed a correlation value of $0.25(\mathrm{p}<0.01)$. In the scale's validity, Cronbach's alpha value was estimated to be 0.797 , and the split-half reliability coefficient was estimated to be 0.771 .

Conclusion Obsession with the COVID-19 Scale can be used as a suitable measurement tool in research.

Keywords Obsession; COVID-19; Psychometrics; SARS-CoV-2
${ }^{1}$ Department of Psychology, Faculty of Social Sciences and Economics, $\mathrm{Bu}$ Ali Sina University, Hamedan, Iran

${ }^{2}$ Department of Psychology, Faculty of Basic Sciences and Literature, Avrasya University, Trabzon, Turkey ${ }^{3}$ Department of Counseling Psychology, Faculty of Higher Education, Trabzon University, Trabzon, Turkey

\section{*Correspondence}

Address: Bu-Ali Sina University, Shahid Mostafa Ahmadi Roshan Street, Hamedan. Postal Code: 6516738695.

Phone: +98 (83) 43752217

Fax: +98 (83) 43752217 kambiz.karimi90@gmail.com

\section{Article History}

Received: October 16, 2020

Accepted: December 07, 2020

ePublished: March 03, 2021

\section{I T A T I O N L I N K S}

[1] Using psycho neuro immunity against COVID-19 [2] The Psychology of pandemics: Preparing for the next global outbreak of infectious disease [3] Impact of the COVID-19 pandemic on mental health and quality of life among local residents in Liaoning Province, China: A cross-sectional study [4] Vicarious traumatization in the general public, members, and non-members of medical teams aiding in COVID-19 control [5] A nationwide survey of psychological distress among Chinese people in the COVID-19 epidemic: implications and policy recommendations [6] Impact of the COVID-19 pandemic on adult mental health [7] A nationwide survey of psychological distress among Italian people during the COVID-19 pandemic: Immediate psychological responses and associated factors [8] Immediate psychological responses and associated factors during the initial stage of the 2019 coronavirus disease (COVID-19) epidemic among the general population in China [9] Thinking Too much about the novel coronavirus, the link between persistent thinking about COVID-19, SARS-CoV-2 anxiety and trauma effects [10] Differences and similarities between obsessive intrusive thoughts and worry in a non-clinical population: Study 2 [11] Psychometric properties of the Beck Depression Inventory: Twenty-five years of evaluation [12] How much "thinking" about COVID-19 is clinically dysfunctional? [13] Factorial validity of the Urdu version of the obsession with COVID-19 scale: Preliminary investigation using a university sample in Pakistan [14] Measuring anxiety related to COVID-19: A Turkish validation study of the coronavirus anxiety scale [15] Review of A Beginner's Guide to Structural Equation Modeling (4th ed.) [16] Linguistic validation manual for health outcome assessments [17] An empirical approach for validation of inter-rater reliability of identified candidate aspects [18] The fear of COVID-19 scale: Development and initial validation [19] Iranian positive thinking scale (IPTS): Development and standardization 
اين موضوع، يِيامدهاى روانشناختى را بهدنبال خواهد داشت]11]. از

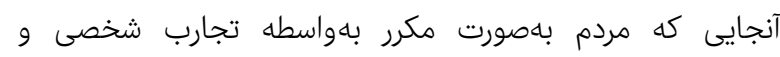

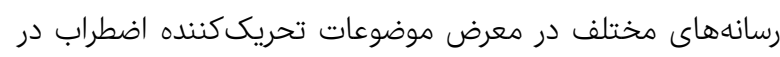

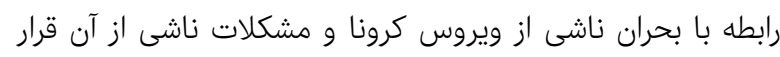

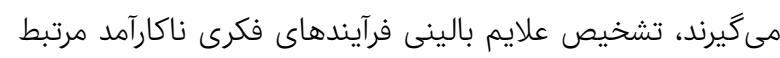

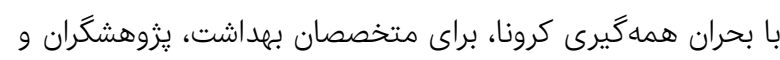

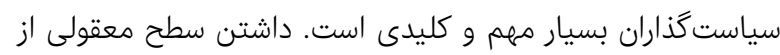

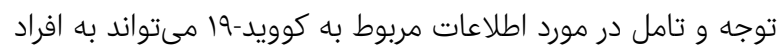
در درازمدت كمك نمايد تا در طول اين بحران، ايمنى مناسب خورد

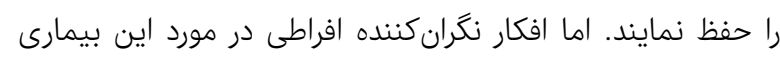

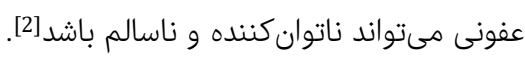

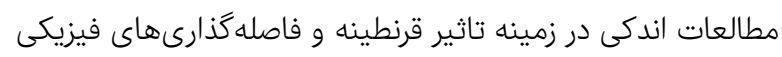

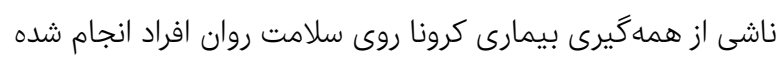

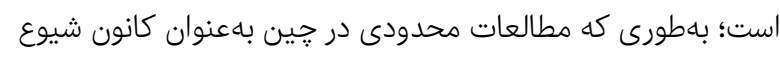

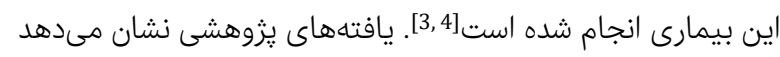

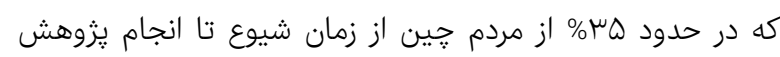

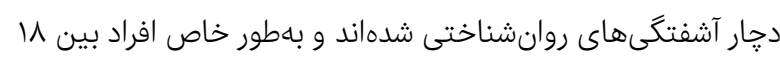

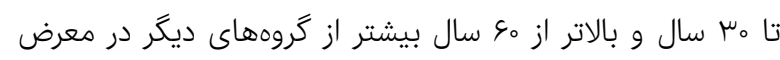

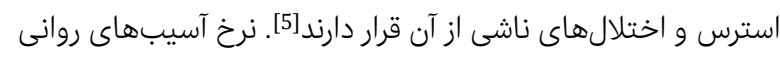

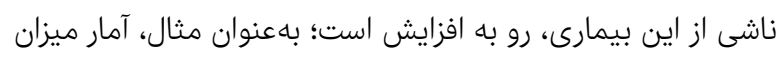

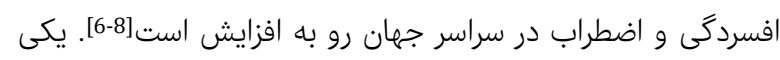

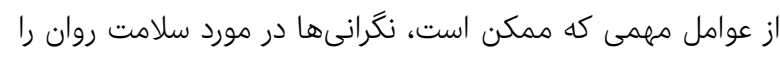

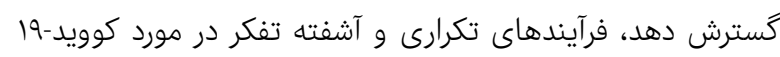

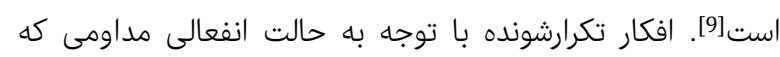

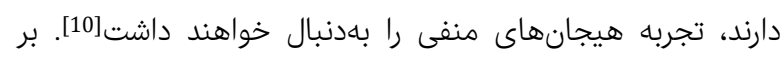

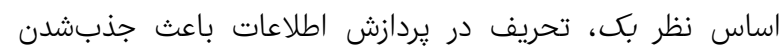

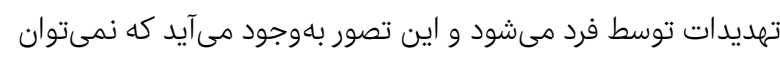

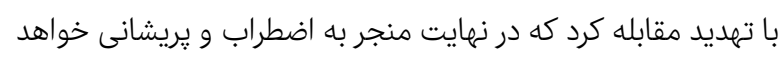

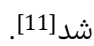

با توجه به جريان مداوم كزارشهاى خبرى در مورد كوويد-19 و و

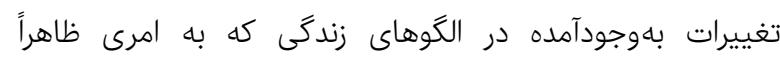

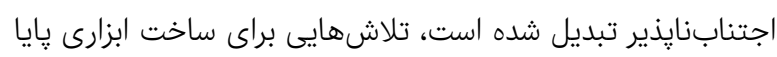

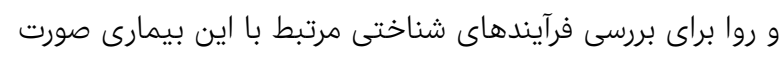

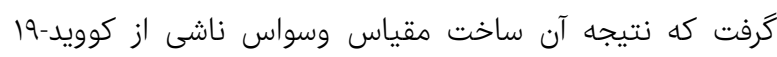
(Obsession with the COVID-19 scale)

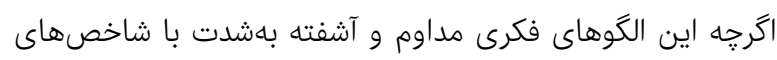

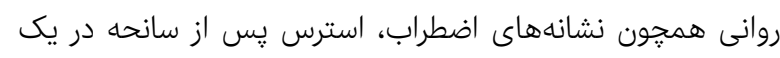

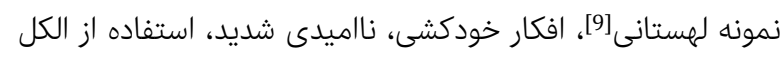

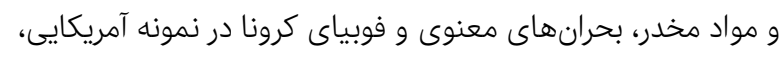

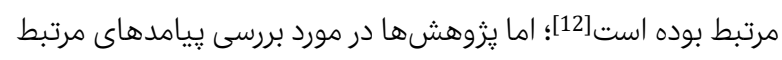

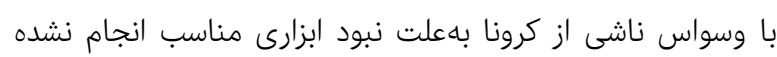

است.

لى، براى پاسخ به اين يرسش كه ميزان فكركردن در مورد بيمارى

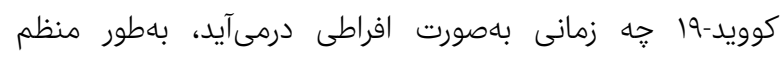

دوره rا، شماره F، پاييز وجسا
روايى و گايايى مقياس وسواس ناشى از كوويد-19

ابوالقاسم يعقوبى PhD

كروه روانشناسى، دانشكده علوم اجتماعى و اقتصاد، دانشكاه بوعلى سينا، همدان، ايران

شيوا صايقى ممقانى PhD

كروه روانشناسى، دانشكده علوم بايه و ادبيات، دانشگاه اوراسيا، ترابزون، تركيه

PhD محمد بالانجى

كروه روانشناسى مشاوره، دانشكده علوم تربيتى، دانشكاه ترابزون، ترابزون،

تركيه

PhD "كامبيز كريمى

كروه روانشناسى، دانشكده علوم اجتماعى و اقتصاد، دانشكاه بوعلى سينا، همدان، ايران

جكيده

اهداف: گسترش كوويد-19 در سراسر جهان سلامت عمومى را به خالش كشيده و

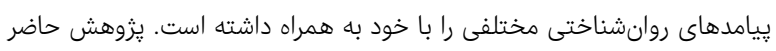

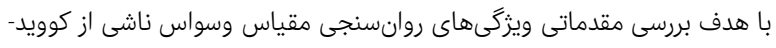
إنجام شد.

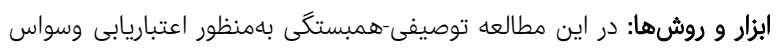

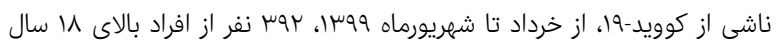

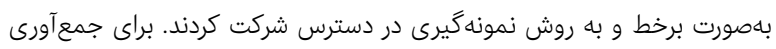

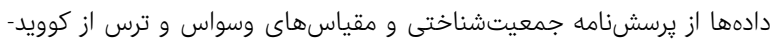

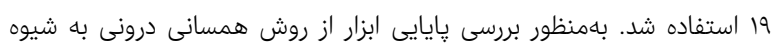

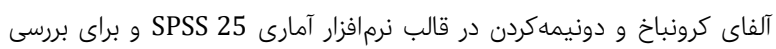

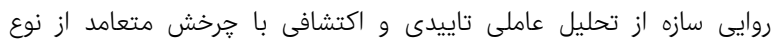

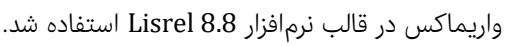

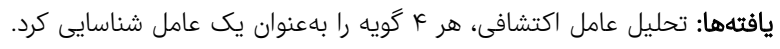

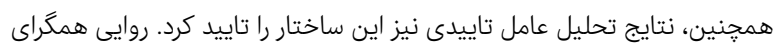

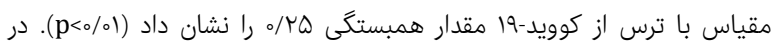

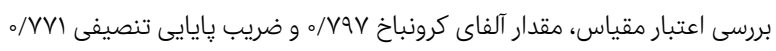

برآورد شد.

نتيجهكيرى: مىتوان مقياس وسواس ناشى از كوويد-19 را بهنوانوان ابزار

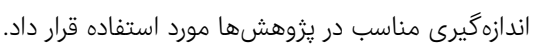

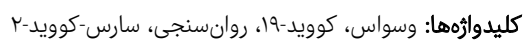

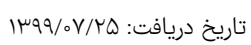

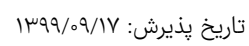

kambiz.karimi90@gmail.com : ناريخ بذينده مسئون:

مقدمه

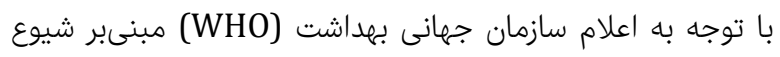

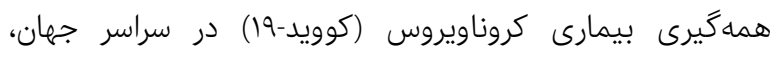

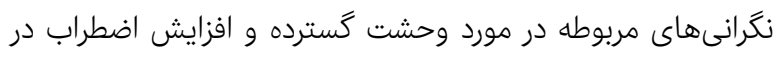

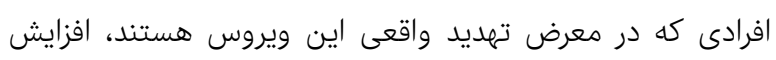

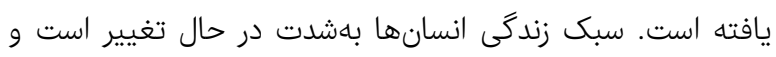

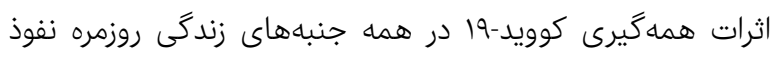

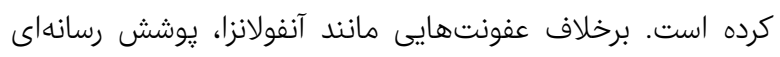

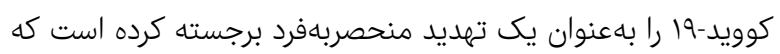




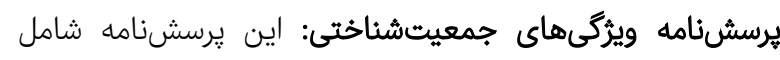

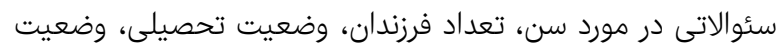

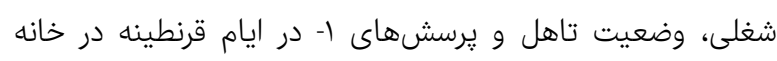

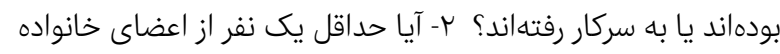

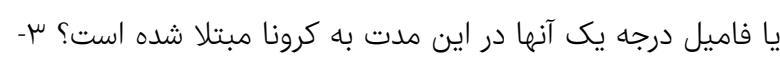

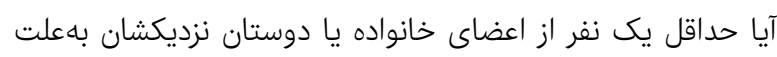

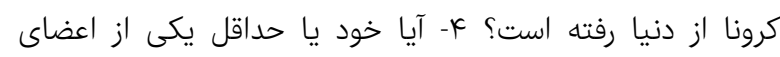

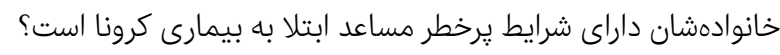

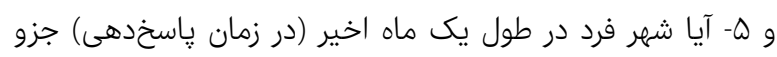

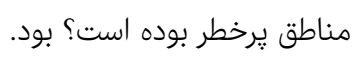
مقياس وسواس ناشى از كوويد-19: نسخه اوليه اين مقياس توسط

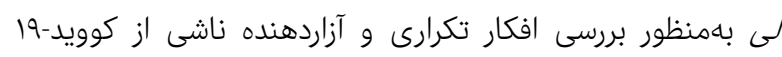

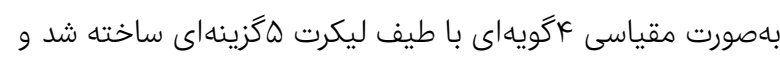

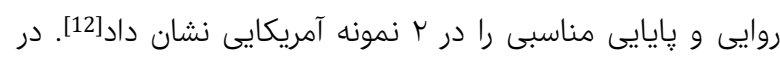

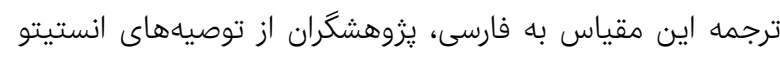

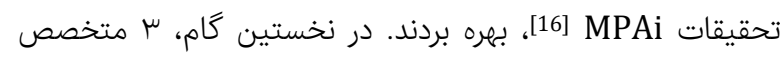

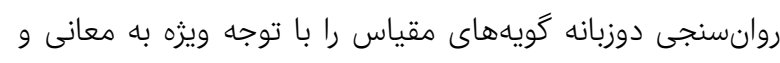

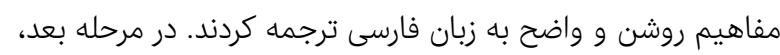

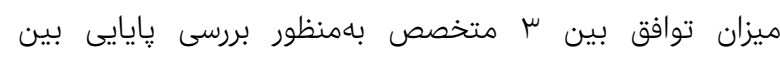

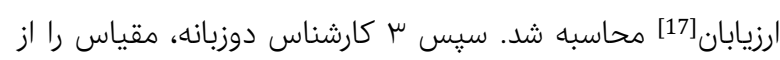

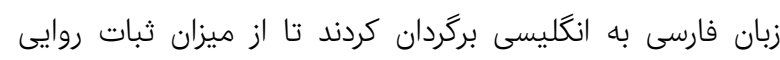

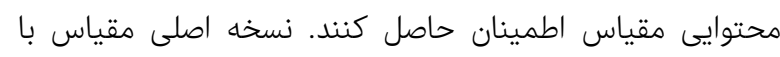

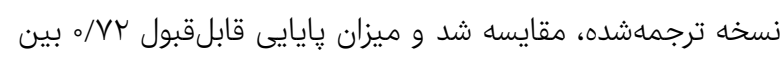

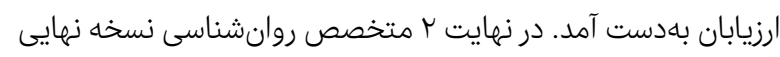

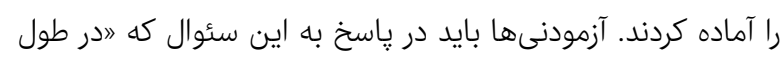

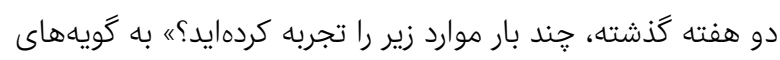

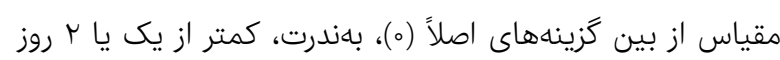

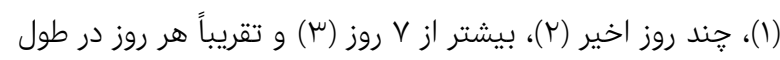

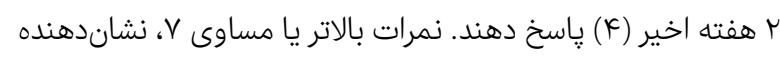

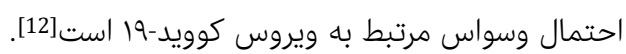

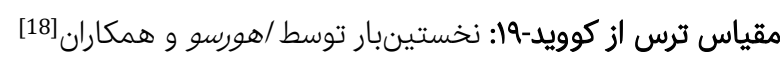

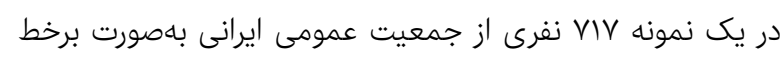

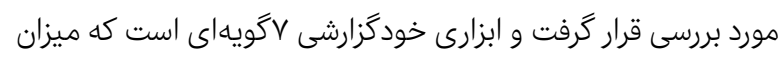

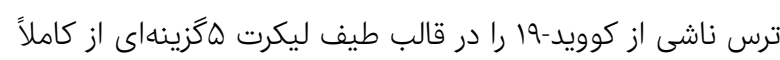

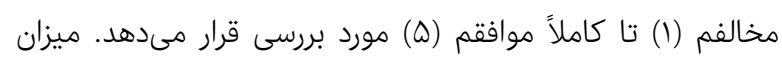

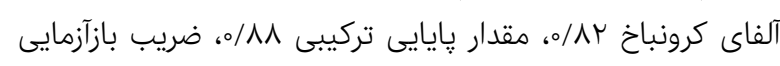

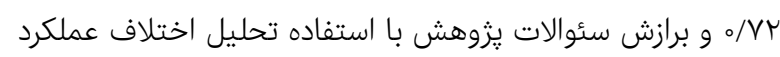

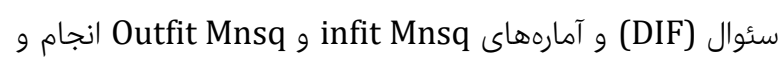

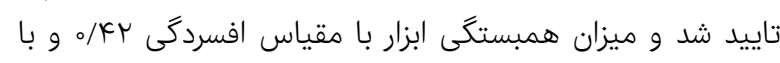

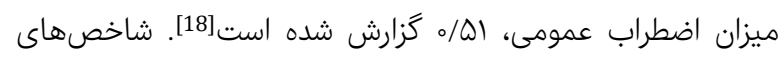

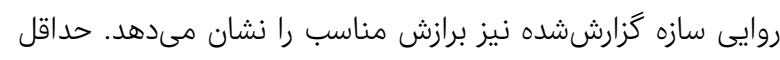

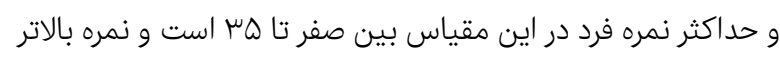

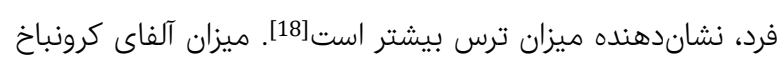

فرآيندهاى فكرى درباره كرونا و خُكونكى ارتباط آن با اختلال در

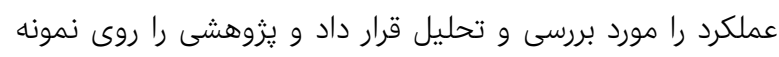

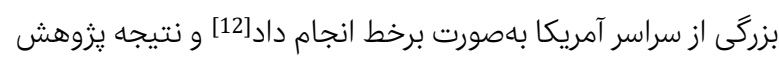

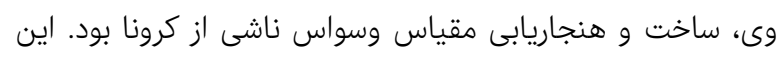

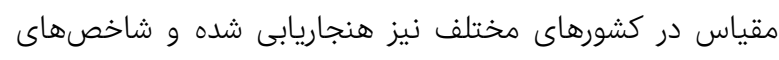

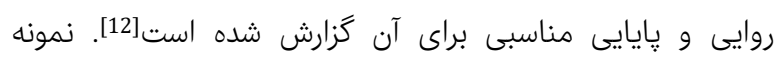

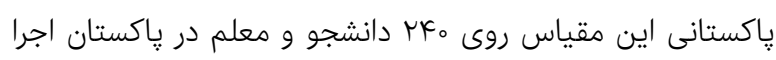

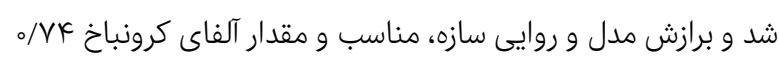

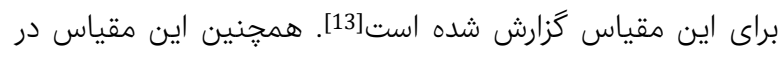

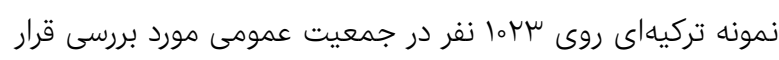

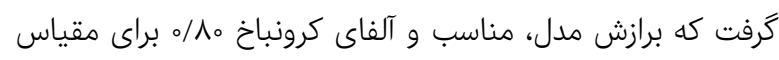
كزارش شده است كن برازت مدل،

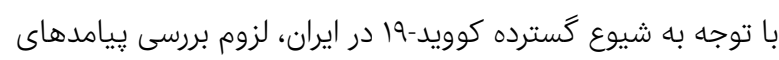

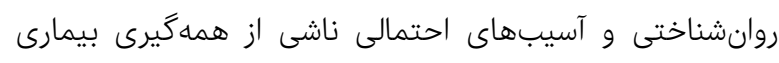

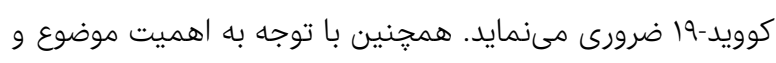

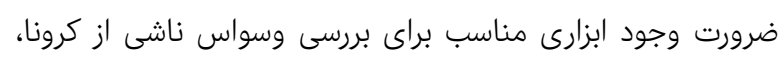

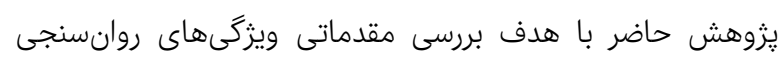

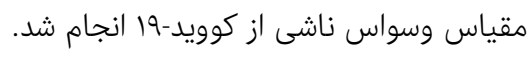

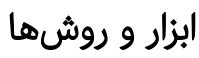

اين يزوهش از نظر هدف، كاربردى و از نظر روش إشناسى در زمره

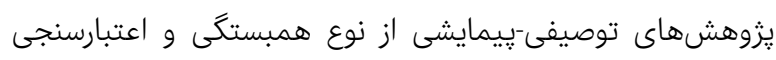

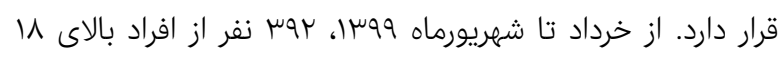

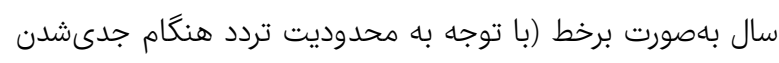

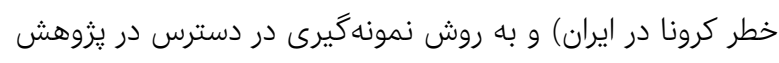

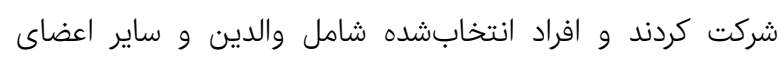

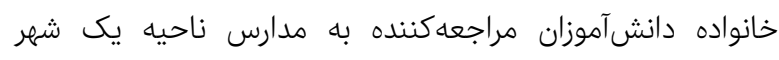

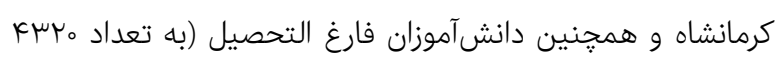

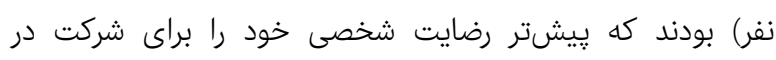

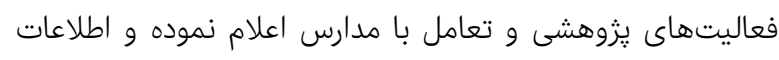

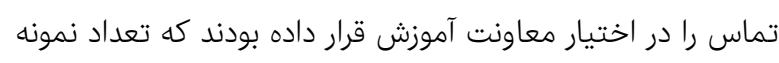

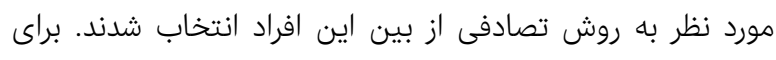

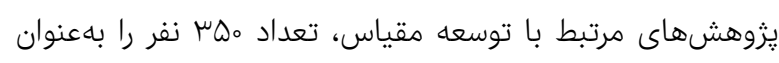

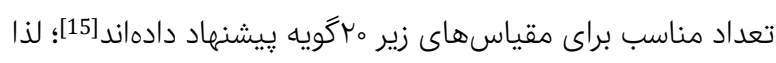

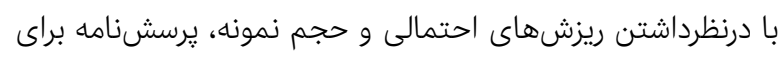

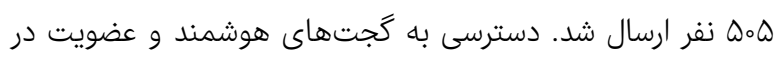

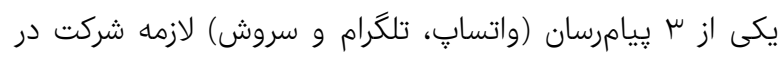

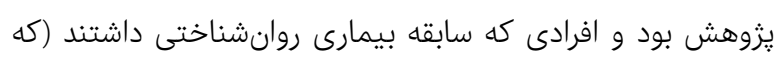

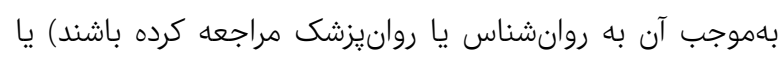

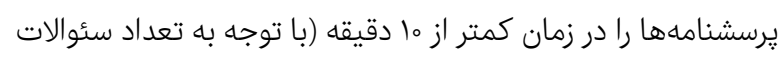

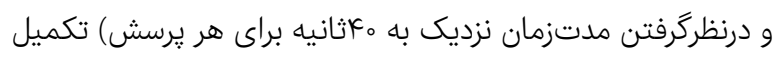
كرده بودند، از مطالعه كنار كذاشته شدند.

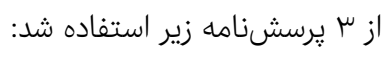


جدول () نتايج فراوانى و درصد فراوانى آزمودنىها از نظر متغيرهاى

جمعيتشناختى جلتول

\begin{tabular}{|c|c|c|}
\hline درود - مد & 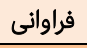 & كروه \\
\hline & & سن \\
\hline $19 / V$ & VE & \1 تا FF سال \\
\hline$r V / V$ & $10 \mathrm{~V}$ & هr تا •ـ سال \\
\hline ( & w。 & 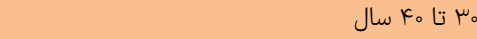 \\
\hline $18 / 1$ & $8 \Delta$ & 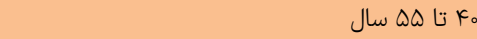 \\
\hline \multirow[t]{2}{*}{$r / l$} & $\wedge$ & ه سال به بالا \\
\hline & & تعداد فرزندان \\
\hline$\Delta \omega / \Gamma$ & שוץ & بدون فرزند \\
\hline $1 V / 9$ & 99 & يك \\
\hline$r M / \Lambda$ & $\Delta F$ & $r$ \\
\hline$\mu / k$ & Ir & $\mu$ \\
\hline $1 / \mu$ & $\Delta$ & k \\
\hline \multirow[t]{2}{*}{$\circ / 0$} & r & بيش از F \\
\hline & & وضعيت تحصيلى \\
\hline$r / \Lambda$ & 11 & زير دييلم \\
\hline 10 & $\Delta \wedge$ & دييلم \\
\hline$\mu / q$ & 10 & 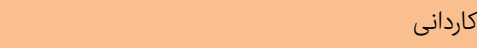 \\
\hline $\mathrm{kq} / \mathrm{V}$ & 194 & كارشناسى \\
\hline$r / Q$ & ᄉ & كارشناسى ارشد \\
\hline \multirow[t]{2}{*}{ V } & rV & 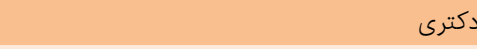 \\
\hline & & وضعيت شغلى \\
\hline $19 / 9$ & VV & 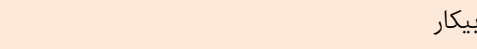 \\
\hline$\mu \omega / \Lambda$ & Iru & 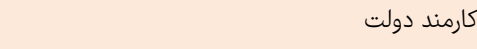 \\
\hline$r e / 4$ & $10 \mathrm{r}$ & 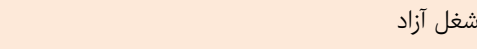 \\
\hline $1 / 4$ & 4 & بازنشسته \\
\hline \multirow[t]{2}{*}{$1 \varepsilon / \mu$} & q & 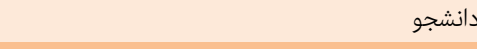 \\
\hline & & وضعيت تاهل \\
\hline $\mathrm{kV} / \mathrm{G}$ & WN & مجرد \\
\hline$k \varepsilon / 1$ & IVA & متاهل \\
\hline$\circ / V$ & $\mu$ & همسر فوتشده (همراه با خانواده) \\
\hline$r / V$ & 10 & همسر فوتشده (زندگى مستقل از خانواده) \\
\hline$r / V$ & 10 & از همسر جداشده (همراه با خانواده) \\
\hline \multirow[t]{2}{*}{$\circ / \gamma$} & 1 & از همسر جداشده (زندگى مستقل از خانواده) \\
\hline & & در ايام قرنطينه در خانه بودهاند يا به سركار رفتهاند؟ \\
\hline $8 \Lambda / 1$ & r & 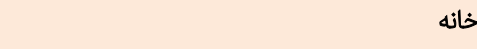 \\
\hline$\mu / / q$ & سKr & 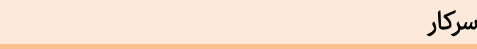 \\
\hline
\end{tabular}

آيا حداقل يك نفر از اعضاى خانواده يا فاميل درجه يك آنها در اين مدت به كرونا

مبتلا شده است؟ حلى ئك

$\Delta 1 / \mu \quad 191$

KN/V IMN

آيا حداقل يك نفر از اعضاى خانواده يا دوستان نزديكشان بهعلت كرونا از دنيا رفته

خله

آيا خود يا حداقل يكى از اعضاى خانوادهشان داراى شرايط برخطر مساعد ابتلا به

بيمارى كرونا است؟

$\mu \mu / V \quad 1 \mu$

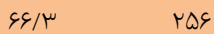

آيا شهر فرد در طول يك ماه اخير (در زمان ياسخدهى) جزو مناطق يرخطر بوده است؟ بله $\mu \Delta / r \quad$ I خير

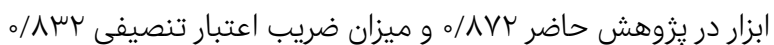

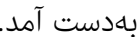

تجزيهوتحليل دادهها در دو بخش توصيفى (فراوانى و ميانكين) و إنداطئ

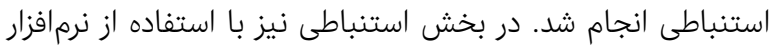

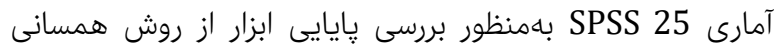

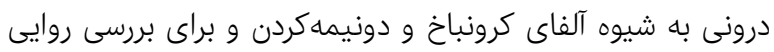

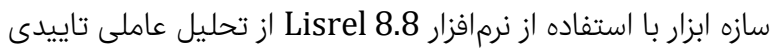

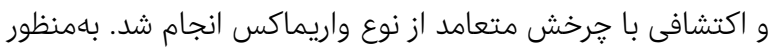

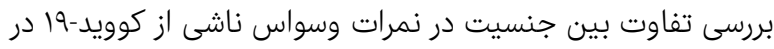

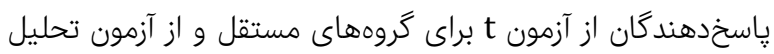

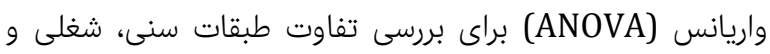

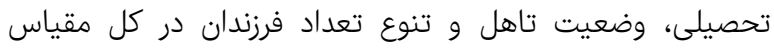

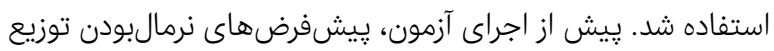

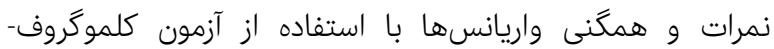

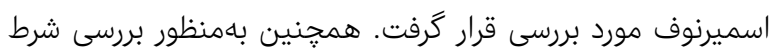

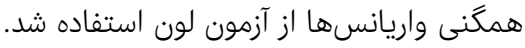

\section{يافتهها}

از بوس برسشنامه، 9 مورد بهصورت كامل تكميل نشده بود و از

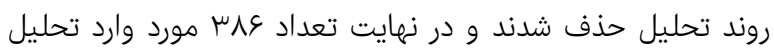

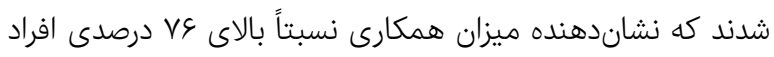

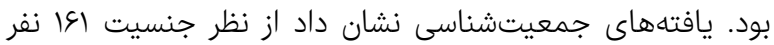
(\% مرد و Fl/V)

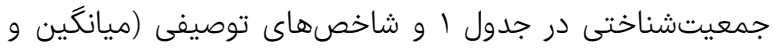

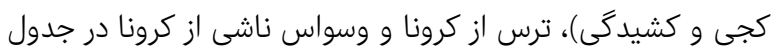

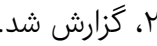
نتايج آزمون كلموكروف-اسميرنوف نشان داد كه توزيع دادهها نرمال

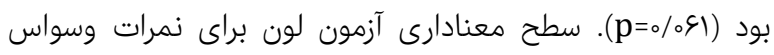

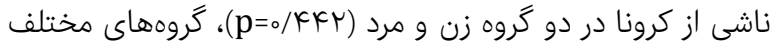
سنى (

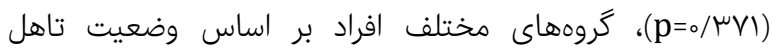

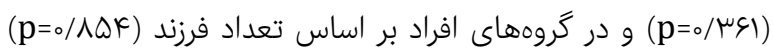

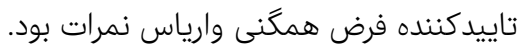

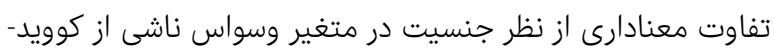

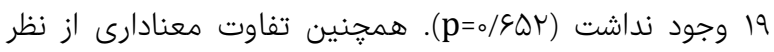

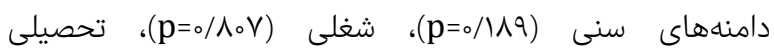

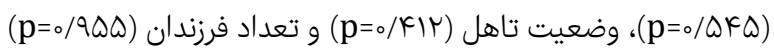
در وسواس ناشى از كوويد-19 وجود نداشت.

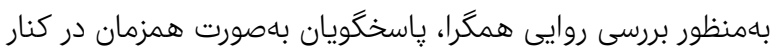

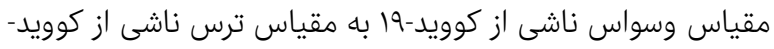

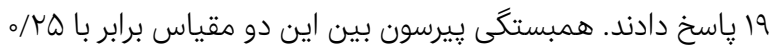

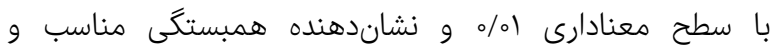
تاييدكننده روايى همكرايى اين مقياس بود. 


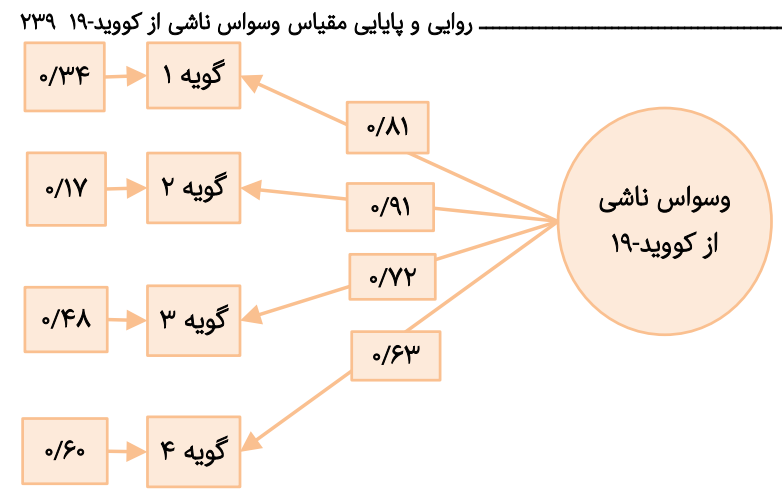

نمودار Y) مدل تكعاملى مقياس وسواس ناشى از كوويد-19 در حالت تخمين

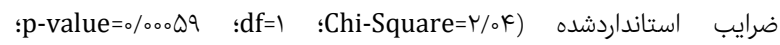

(RMSEA=o/oو

مقدار ضريب آلفاى كرونباخ براى مقياس وسواس ناشى از كوويد-

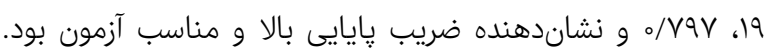

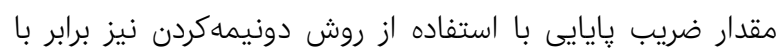

1/VVI

بحث

كَسترش كوويد-19 در سراسر جهان سلامت عمومى را به קالش

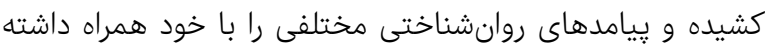

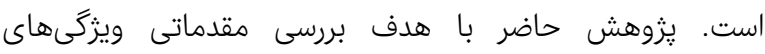
روانسنجى مقياس وسواس ناشى از كوويد-19 انجام شد. بهاطور كلى دئى

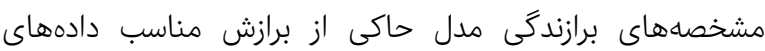

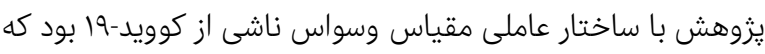

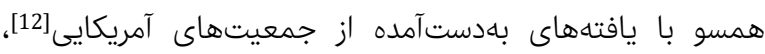

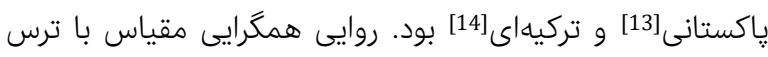

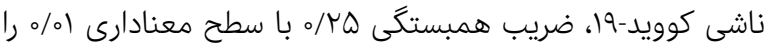

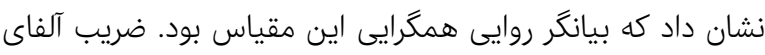

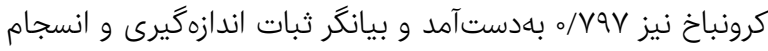

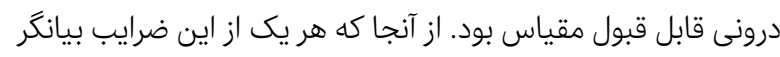

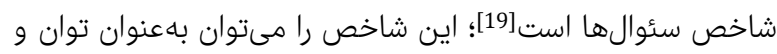

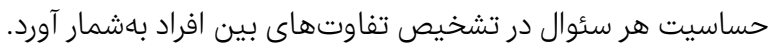

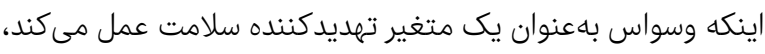

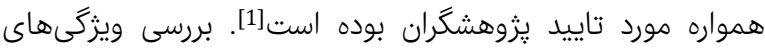
روانسنجى مقياس وسواس ناشى از كوويد-19 در جامعه ايرانى بردي

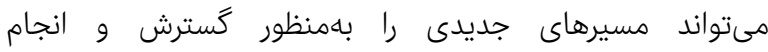

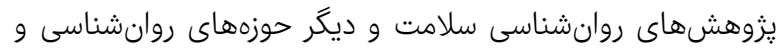

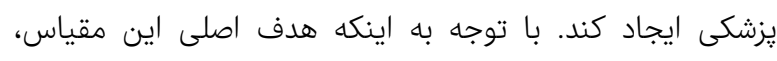

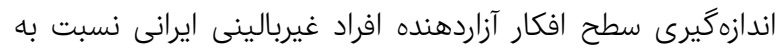

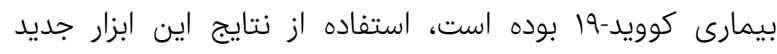

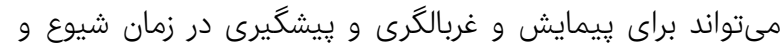

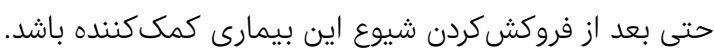

جدول r) شاخصهاى توصيفى متغيرهاى يزوهش

\begin{tabular}{|c|c|c|c|}
\hline كشيدكى & جولگى & ميانكين & متغير \\
\hline $1 / 9 \Lambda$ & $1 / \mu r$ & $\mu / \varepsilon \mid \pm \psi / \Delta \varphi$ & وسواس ناشى از كرونا \\
\hline$-\circ / \mu q$ & $0 / r_{0}$ & $|N / \varepsilon| \pm \varepsilon / \mu_{0}$ & ترس از كرونا \\
\hline
\end{tabular}

نتايج اوليه تحليل مولفههاى اصلى نشان داد كه تمامى سئوالات

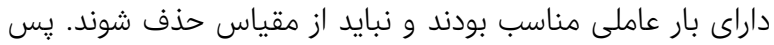

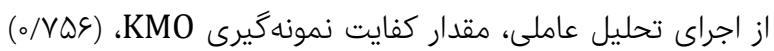

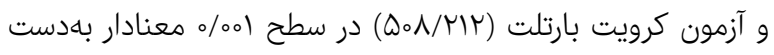
آهد؛ بنابراين با توجه به بالابودن مقدار KMO

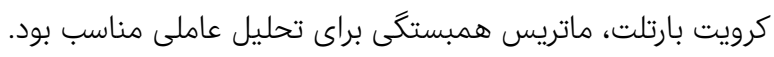

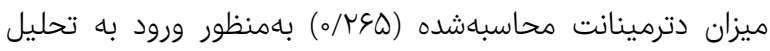

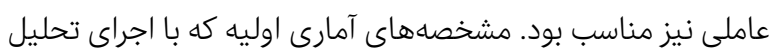

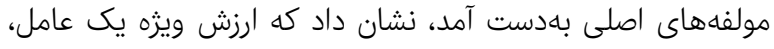

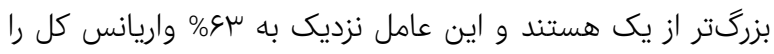

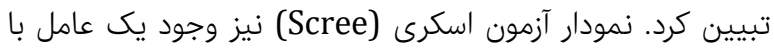

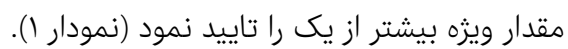

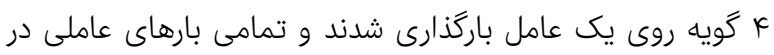

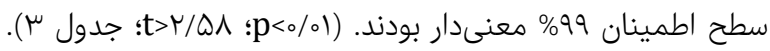

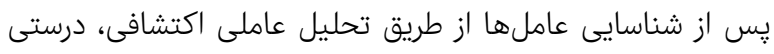

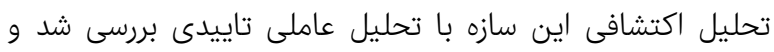

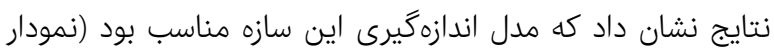

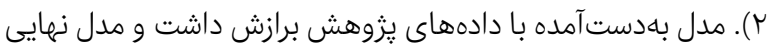

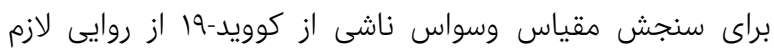
برخوردار بود.

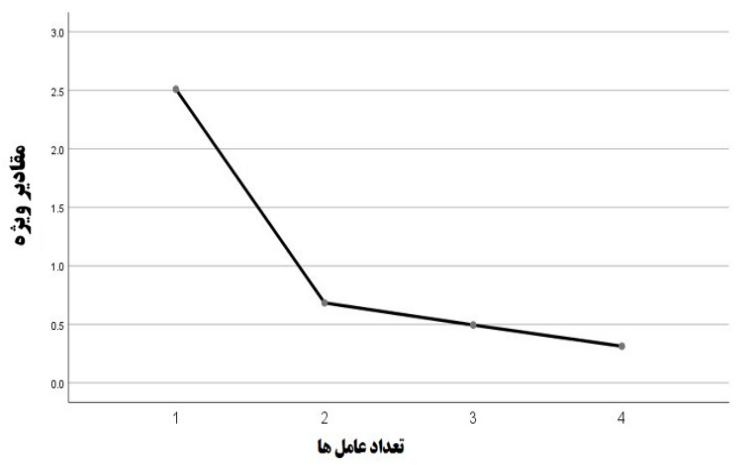

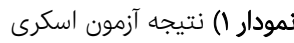

\begin{tabular}{|c|c|c|}
\hline t-value & 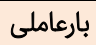 & كويهما \\
\hline N/.0 & ס/Arm & كرونا كرفته باشم. \\
\hline T/MT & $\circ / \wedge \Delta V$ & 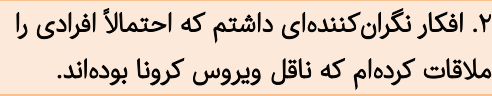 \\
\hline $10 / \Delta 9$ & $\circ / \Lambda_{0} \mu$ & ץ. نمىتوانستم به ويروس كرونا فكر نكنم. \\
\hline $1 \% / \% k$ & $0 / 8 \mathrm{Vr}$ & F. در مورد بيمارى كرونا خواب ديدم. \\
\hline
\end{tabular}


5- Qiu J, Shen B, Zhao M, Wang Z, Xie B, Xu Y. A nationwide survey of psychological distress among Chinese people in the COVID-19 epidemic: implications and policy recommendations. Gen Psychiatry. 2020;33(2): e100213. 6- Haider II, Tiwana F, Tahir SM. Impact of the COVID-19 pandemic on adult mental health. Pak J Med Sci. 2020;36(COVID19-S4):S90-4

7- Mazza C, Ricci E, Biondi S, Colasanti M, Ferracuti S, Napoli C, et al. A nationwide survey of psychological distress among Italian people during the COVID-19 pandemic: Immediate psychological responses and associated factors. Int J Environ Res Public Health. 2020;17(9):3165.

8- Wang C, Pan R, Wan X, Tan Y, Xu L, Ho CS, Ho RC. Immediate psychological responses and associated factors during the initial stage of the 2019 coronavirus disease (COVID-19) epidemic among the general population in China. Int J Environ Res Public Health. 2020;17(5):1729.

9- Skalski S, Uram P, Dobrakowski P, Kwiatkowska A. Thinking Too much about the novel coronavirus, the link between persistent thinking about COVID-19, SARS-CoV2 anxiety and trauma effects. Curr Issues Personal Psychol. 2020;8(3):169-74.

10- Langlois F, Freeston MH, Ladouceur R. Differences and similarities between obsessive intrusive thoughts and worry in a non-clinical population: Study 2. Beh Res Ther. 2000;38(2):175-89.

11- Beck AT, Steer RA, Carbin MG. Psychometric properties of the Beck Depression Inventory: Twenty-five years of evaluation. Clin Psychol Rev. 1988;8(1):77-100.

12- Lee SA. How much "thinking" about COVID-19 is clinically dysfunctional?. Brain Behav Immun. 2020;87:97-8

13- Ashraf F, Lee SA, Crunk A E. Factorial validity of the Urdu version of the obsession with COVID-19 scale: Preliminary investigation using a university sample in Pakistan. Death Stud. 2020;1-6.

14- Evren C, Evren B, Dalbudak E, Topcu M, Kutlu N. Measuring anxiety related to COVID-19: A Turkish validation study of the coronavirus anxiety scale. Death Stud. 2020;1-7.

15- Stegmann RB. Review of A Beginner's Guide to Structural Equation Modeling (4th ed.), by Randall E. Schumacker \& Richard G. Lomax. Struct Equ Model Multidisciplinary J. 2017;24(3):475-7.

16- Acquadro C, Conway K, Giroudet C, Mear I. Linguistic validation manual for health outcome assessments. Lyon: Mapi Institute; 2012.

17- Aravindan JS, Vivekanandan K. An empirical approach for validation of inter-rater reliability of identified candidate aspects. Int J Comput Sci Eng. 2017;5(5):94100.

18- Ahorsu DK, Lin CY, Imani V, Saffari M, Griffiths MD, Pakpour AH. The fear of COVID-19 scale: Development and initial validation. Int J Ment Health Addict. 2020;1-9.

19- Khodayari Fard M, Ghobari Bonab B, Akbari Zardkhaneh S, Zamonpour EA, Derakhshan M, Musavi SA. Iranian positive thinking scale (IPTS): Development and standardization. J Appl Psychol Res. 2015;5(4):103-30. [Persian]
كوتاهبودن مقياس مورد نظر و سهولت اجراى آن مىتواند براى يزوهشهايى كه با قصد بررسى روابط بين اين سازه و متغيرهاى مورد

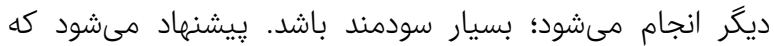

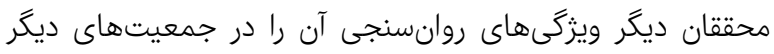

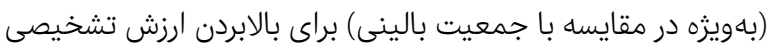

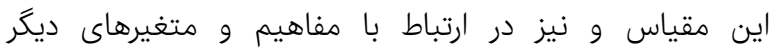
روانشناختى، اجتماعى و يزشكى بررسى كنند. از آنجايى كه در زمان

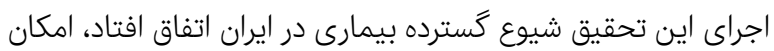

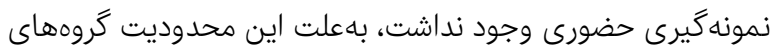

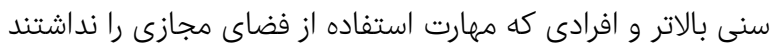

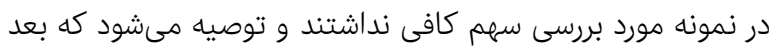

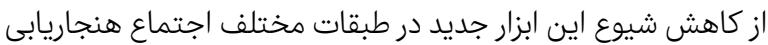

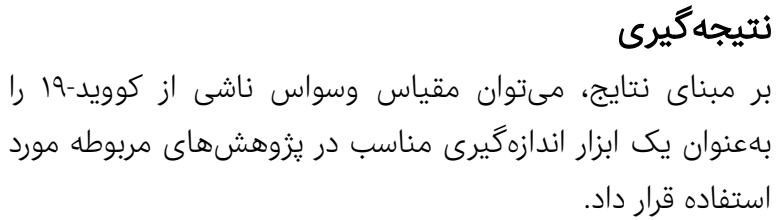

تشكر و قدردانى: يزّوهشكران بر خود لازم مىدانند از تمامى افرادى كه در

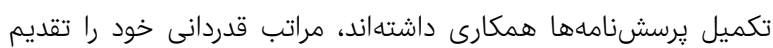

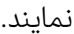

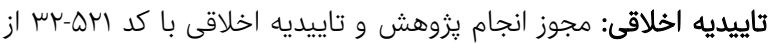

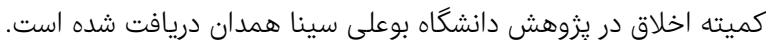

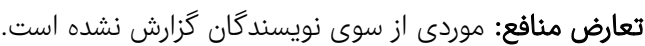

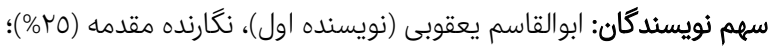

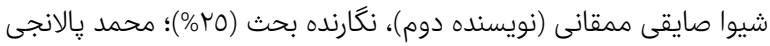

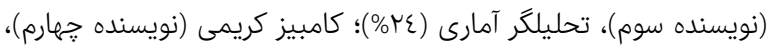

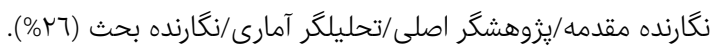

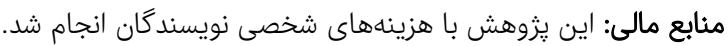

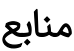

1- Kim SW, Su KP. Using psycho neuro immunity against COVID-19. Brain Behav Immun. 2020;87:4-5.

2- Taylor S. The Psychology of pandemics: Preparing for the next global outbreak of infectious disease. Newcastle upon Tyne: Cambridge Scholars Publishing; 2019.

3- Zhang Y, Ma ZF. Impact of the COVID-19 pandemic on mental health and quality of life among local residents in Liaoning Province, China: A cross-sectional study. Int J Environ Res Public Health. 2020;17(7):2381.

4- Li Z, Ge J, Yang M, Feng J, Qiao M, Jiang R, et al. Vicarious traumatization in the general public, members, and nonmembers of medical teams aiding in COVID-19 control. Brain Behav Immun. 2020;88:916-9. 